ARTICLE

Received 19 Feb 2014 | Accepted 12 Nov 2014 | Published 23 Dec $2014 \quad$ DOl: 10.1038/ncomms6832

\title{
Using ultrashort optical pulses to couple ferroelectric and ferromagnetic order in an oxide heterostructure
}

\author{
Y.M. Sheu', S.A. Trugman', L. Yan¹, Q.X. Jia', A.J. Taylor ${ }^{1} \&$ R.P. Prasankumar ${ }^{1}$
}

A new approach to all-optical detection and control of the coupling between electric and magnetic order on ultrafast timescales is achieved using time-resolved second-harmonic generation ( $\mathrm{SHG}$ ) to study a ferroelectric (FE)/ferromagnet (FM) oxide heterostructure. We use femtosecond optical pulses to modify the spin alignment in a $\mathrm{Ba}_{0.1} \mathrm{Sr}_{0.9} \mathrm{TiO}_{3}$ (BSTO)/ $\mathrm{La}_{0.7} \mathrm{Ca}_{0.3} \mathrm{MnO}_{3}$ (LCMO) heterostructure and selectively probe the ferroelectric response using SHG. In this heterostructure, the pump pulses photoexcite non-equilibrium quasiparticles in LCMO, which rapidly interact with phonons before undergoing spin-lattice relaxation on a timescale of tens of picoseconds. This reduces the spin-spin correlations in LCMO, applying stress on BSTO through magnetostriction. This then modifies the FE polarization through the piezoelectric effect, on a timescale much faster than laser-induced heat diffusion from LCMO to BSTO. We have thus demonstrated an ultrafast indirect magnetoelectric effect in a FE/FM heterostructure mediated through elastic coupling, with a timescale primarily governed by spin-lattice relaxation in the FM layer.

\footnotetext{
${ }^{1}$ Center for Integrated Nanotechnologies, Los Alamos National Laboratory, MS K771, Los Alamos, New Mexico 87545, USA. Correspondence and requests for materials should be addressed to Y.M.S. (email: yumiinsheu@gmail.com) or R.P.P. (email: rpprasan@lanl.gov).
} 
$\mathrm{M}$ agnetoelectric (ME) multiferroics have attracted much recent attention, largely due to the scarcity of naturally occurring materials in which magnetic and electric order coexist ${ }^{1-4}$. However, the microscopic mechanisms underlying ME coupling between these order parameters have been difficult to unravel, hindering the development of single-phase multiferroics with strong ME coupling at useful temperatures ${ }^{1,2,5}$. Artificial multiferroic composites ${ }^{6-9}$ offer an appealing alternative, in which the ME coupling can be engineered through proper choice of the interface geometry and constituent materials ${ }^{4}$. The most well-known approach for achieving strong ME coupling in these systems uses strain to indirectly couple ferroelectric (FE) and ferromagnetic (FM) order in two-phase-layered composites ${ }^{5,7,10}$. In these composites, the ME effect is essentially a product of the magnetostrictive effect in the FM layer and the piezoelectric effect in the FE layer ${ }^{5,10-13}$. A magnetic (B) field is used to modify the spin alignment in the FM layer, which changes the lattice constant through magnetostriction. This in turn applies stress on the FE layer and modifies the FE polarization through the piezoelectric effect $^{12-14}$, significantly increasing the ME coupling as compared with single-phase multiferroics ${ }^{5,10}$. Several reviews describing the $\mathrm{ME}$ effect in these composites have been recently published ${ }^{5,10,12,15}$.

Despite these impressive advances, an important aspect of multiferroics has received relatively little attention: namely, their dynamic properties ${ }^{2,3,10}$, which will impact many of their potential applications. In fact, the ultimate timescales limiting the speed of ME coupling in these materials, as well as the physical processses governing them, remain relatively unexplored. Femtosecond optical pulses are particularly useful in this regard $^{16-31}$, since they can be used to both interrogate and control the ME response in a non-contact manner on ultrafast timescales, much faster than by directly applying magnetic or electric fields. Furthermore, specific order parameters can be directly accessed using time-resolved second-harmonic generation (TR-SHG), making it especially attractive for studying both magnetically ${ }^{20}$ and orbitally ordered ${ }^{32}$ materials, as well as multiferroics ${ }^{18}$ and interfacial properties of novel layered materials ${ }^{19}$ in the time domain.

Here, we use TR-SHG to explore and optically manipulate the coupling between FE and FM order in an oxide heterostructure for the first time, inspired by the strain/stress-based approach described above. By separating the different contributions to the TR-SHG signal in the time domain, we discovered that the timescale dominating the ME response is governed by demagnetization of the FM layer through spin-lattice relaxation. More specifically, optically perturbing magnetic order in the FM layer imposes lateral stress on the $\mathrm{FE}$ layer through magnetostriction, modifying FE order within tens of picoseconds. This demonstrates that femtosecond optical pulses can be used not only to give insight into the microscopic mechanisms underlying ME coupling in complex oxide heterostructures, but also to manipulate the ME response in these systems on ultrafast timescales.

\section{Results}

Static and TR-SHG experiments. The heterostructure studied here consists of a 50-nm-thick film of $\mathrm{FE} \mathrm{Ba}_{0.1} \mathrm{Sr}_{0.9} \mathrm{TiO}_{3}$ (BSTO) and a 50 -nm-thick film of $\mathrm{FM} \mathrm{La}_{0.7} \mathrm{Ca}_{0.3} \mathrm{MnO}_{3}$ (LCMO), grown on a $\mathrm{MgO}$ substrate; more detail on sample fabrication is given in Methods and in ref. 33. We chose BSTO with this specific composition since lattice strain introduced through heteroepitaxial growth can enhance ferroelectricity and the FE transition temperature $\left(T_{\mathrm{FE}}\right)$, as theoretically predicted ${ }^{34,35}$ and experimentally observed ${ }^{33}$. This coupling between strain and FE order makes it a good candidate for inducing indirect ME effects through elastic coupling with a magnetic material. Similarly, LCMO was chosen since a strong ME effect was previously obtained through magnetostriction under an applied d.c. magnetic field, when it was incorporated into a bilayer structure with lead zirconate titanate $e^{5,14,36}$. The concept behind our experiment is thus to demagnetize LCMO with femtosecond optical pulses, modifying its lattice constant through magnetostriction; the resulting stress on BSTO activates its piezoelectric response, changing FE order and indirectly inducing ME coupling within the demagnetization time.

Photoinduced changes in the static FE polarization of BSTO, $P_{s}$, can be measured using SHG (Fig. 1a,b,d), which is well known to directly probe FE order ${ }^{37-40}$. The SHG polarization generated by the fundamental light fields $E_{j}$ and $E_{k}$ is $P_{i}(2 \omega)=\epsilon_{0} d_{i j k} E_{j}(\omega)$ $E_{k}(\omega)$, where $\omega$ is the frequency and $d_{i j k}$ is the nonlinear optical susceptibility tensor ${ }^{39-41}$. Our BSTO films have $C_{4 \mathrm{v}}$ symmetry and a FE polarization along the sample normal ( $z$ in Fig. 1a) due to the in-plane compressive strain induced by lattice matching to $\mathrm{LCMO}^{33}$. There are only three non-vanishing tensor components for this crystal symmetry: $d_{15}, d_{31}$ and $d_{33}$. By varying the polarization of the incident fundamental and detected SHG light (Fig. 1a) while measuring the SHG intensity $I$ (Fig. 1b), these different components can be extracted, as discussed in Methods.

To optically explore the ME response of our BSTO/LCMO heterostructure, we use the majority of the output laser intensity to photoexcite LCMO (at $1.59 \mathrm{eV}$, far below the BSTO band gap of $\sim 3.3-3.6 \mathrm{eV}$; ref. 33 ) and the remainder to measure the
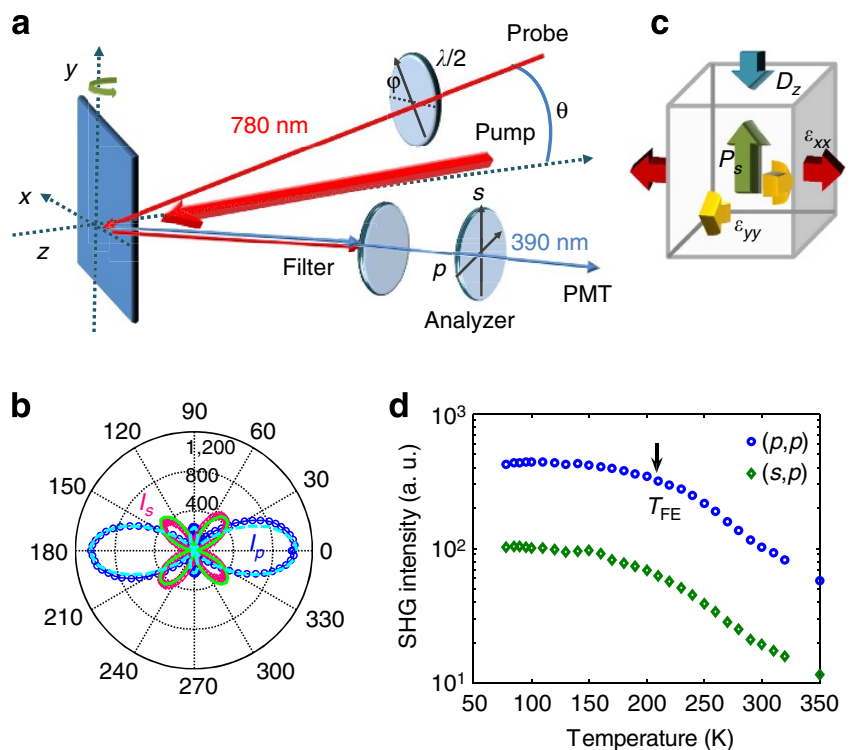

Figure 1 | Experimental set-up, static SHG characterization and piezoelectric response to in-plane stress. (a) Experimental schematic for TR-SHG in reflection. $\theta$ is the angle between the sample normal and the light propagation direction. $\phi$ is the incident polarization of the fundamental light, which is controlled by a half-wave $(\lambda / 2)$ plate. Incident $p$ and $s$ polarizations correspond to $\phi=0^{\circ}\left(180^{\circ}\right)$ and $90^{\circ}\left(270^{\circ}\right)$, respectively. (b) Polar plot of the measured SHG intensity at $10 \mathrm{~K}$. The blue and red symbols are the $p$ - and s-polarized SHG signals, $I_{p}$ and $I_{s}$, respectively, plotted as a function of the incident light polarization. The cyan dashed and green solid lines are numerical fits to $I_{p}$ and $I_{s}$ with $d_{31}=-1, d_{15}=-1.2$ and $d_{33}=7$. (c) Schematic diagram of piezoelectric response along $z$ to applied in-plane stress $\left(\varepsilon_{x x}\right.$ and $\left.\varepsilon_{y y}\right)$. (d) Static temperature-dependent SHG for $(p, p)$ and $(s, p)$ polarization combinations. In LCMO, $T_{C} \sim 240 \mathrm{~K}$, while in BSTO, $T_{\mathrm{FE}} \sim 215 \mathrm{~K}$ (ref. 33), as indicated by the arrow. 
resulting changes in $P_{s}$ using SHG. Photoexcited quasiparticles (unbound electrons and holes) in LCMO will relax through different processes that have been extensively studied using pump-probe spectroscopy ${ }^{42-44}$. Initially, the non-equilibrium quasiparticles rapidly lose energy through electron-phonon ( $\mathrm{e}-\mathrm{ph}$ ) coupling (within $\sim 1 \mathrm{ps}$ ), after which the lattice exchanges energy with the spins through spin-lattice $(s-1)$ coupling on a timescale of tens to hundreds of ps. On longer timescales $(>1 \mathrm{~ns})$, LCMO returns to equilibrium as the remaining energy is dissipated through the substrate. More detail on quasiparticle dynamics in manganites is provided in Supplementary Note 1.

Data analysis. We can relate this to the timescales on which the FE polarization changes in our TR-SHG experiments (Fig. 2). Figure $2 \mathrm{a}$ schematically shows the different processes occurring

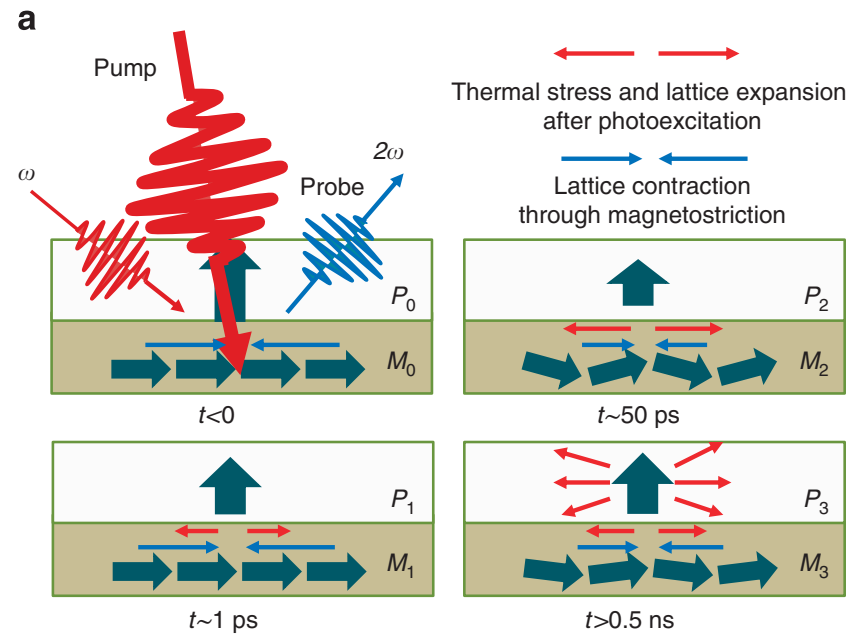

b

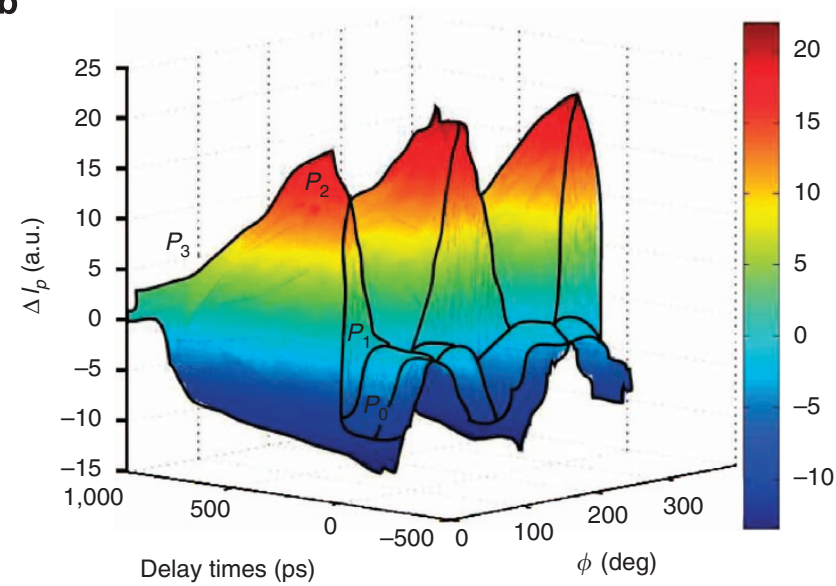

Figure 2 | Photoinduced coupling between ferroelectric and

ferromagnetic order. (a) Schematic diagram of the interplay between the lattice, the magnetization $M$ and the FE polarization $P$ at various pumpprobe delays. After photoexcitation, thermal stress on BSTO (thin red arrows) initially results from e - ph coupling in LCMO within $1 \mathrm{ps}$. Its magnitude gradually increases after relaxation of the lattice contraction in LCMO through magnetostriction (thin blue arrows) on a timescale of $\sim 50$ ps, causing $P$ to decrease. Thermal diffusion from LCMO to both BSTO and the substrate then takes place at longer timescales, $t>0.5 \mathrm{~ns}$, finally dissipating through the substrate. (b) Time-resolved $p$-polarized SHG as a function of the incident light polarization at $10 \mathrm{~K} . P_{\mathrm{O}}-P_{3}$ are the FE polarization responses at different times to changes in the magnetization (arising from $M_{0}-M_{3}$ in a). for different pump-probe delays $t$. Before $t=0$, a small negative change in the $p$-polarized SHG intensity $\left(\Delta I_{p}\right)$ is observed in all polar combinations at low temperatures $\left(P_{0}\right)$, corresponding to residual heating that is not completely dissipated before the arrival of the next pump pulse. This change in $I_{p}$, where the SHG intensity decreases for all incident light polarizations, as more clearly seen in Figs $2 b$ and $3 a$ for negative time delays, is equivalent to increasing the sample temperature in the SHG polar plot of Fig. 1b (or the temperature-dependent plots in Fig. 1d), since before $t=0$ the electron, spin and lattice subsystems share a common temperature.

In contrast, at early times $(t \sim 1-2 \mathrm{ps})$, we observe a polarization-dependent change in $I_{p}\left(P_{1}\right)$, where the SHG intensity both increases and decreases for different polarizations, as shown in Figs $2 \mathrm{~b}$ and 3. This cannot be obtained by simply changing the sample temperature; it is a non-equilibrium effect, occurring before the different subsystems can reach a common temperature. This is due to the elevated temperature of the lattice in LCMO after $\mathrm{e}-\mathrm{ph}$ coupling, causing it to expand, which in turn initiates an in-plane thermal stress $\varepsilon_{x x}$ and $\varepsilon_{y y}$ on BSTO (without changing its temperature) (Fig. 2a). This then initiates an out-of-plane piezoelectric response through $D_{i}=e_{i j k} \varepsilon_{j k}$, where $D_{i}$ is the dielectric displacement and $e_{i j k}$ is the piezoelectric coefficient. In the $C_{4 \mathrm{v}}$ symmetry, $e_{31}$ is non-zero (more detail is given in Methods), linking $\varepsilon_{x x}$ and $\varepsilon_{y y}$ to $D_{z}$ (Fig. 1c) and leading to a photoinduced change in the SHG intensity. It takes $\sim 7 \mathrm{ps}$ for the strain induced by in-plane stress to propagate through the

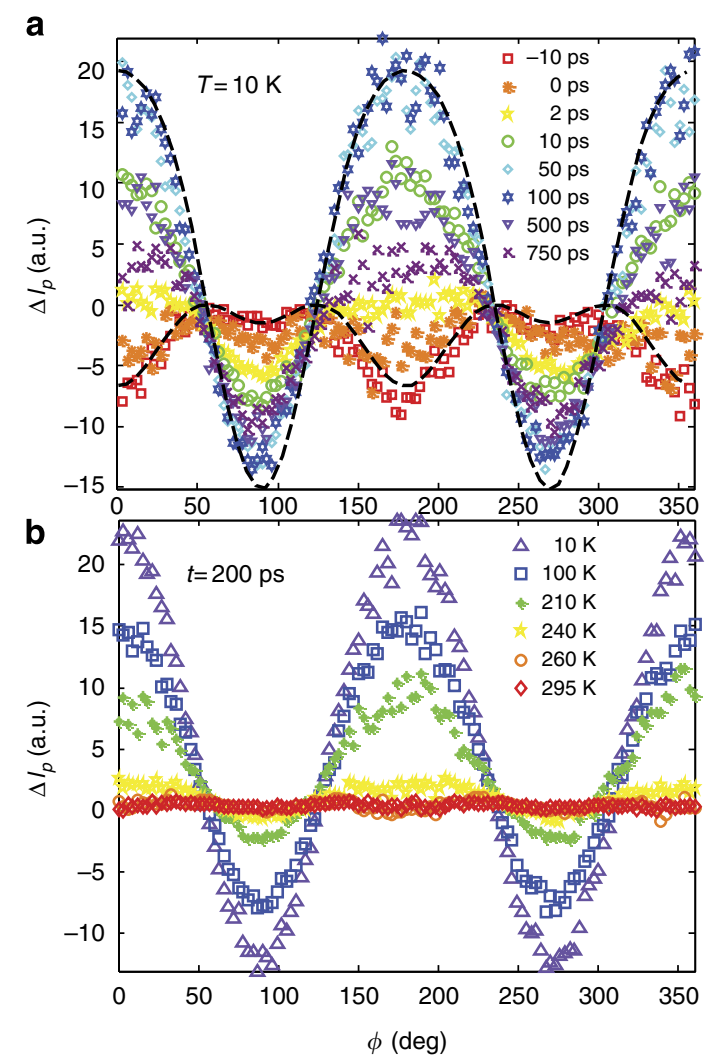

Figure 3 | Evidence for a non-equilibrium, nonlinear origin of the TR-SHG response. (a) Polarization-dependent changes in $I_{p}$ measured at $10 \mathrm{~K}$ for various time delays. The data are plotted as symbols and numerical calculations for $t=-10 ; 50 \mathrm{ps}$ are plotted as dashed lines. Before $t=0$, the change is due to long-lived residual heating from LCMO to BSTO. After $t=0$, the change arises from reductions in $d_{31}$ and $d_{33}$ (more detail is given in Methods). (b) Polarization-dependent changes in $I_{p}$ measured at $t=200 \mathrm{ps}$ for different temperatures. 
BSTO layer (estimated from the speed of sound in BSTO), which agrees relatively well with the timescale of the initial rise in $\Delta I_{p}$, as shown in Fig. 4 (that is, there is no rapid $\sim 1$ ps rise time, unlike the photoinduced change in reflectivity, $\Delta R / R$ ). However, the maximum change in $I_{p}$ (represented by $P_{2}$ ) does not occur on this timescale, but on a much slower timescale of $\sim 50-100$ ps (Figs $2 \mathrm{~b}$ and $3 \mathrm{a}$ ).

We can gain insight into the origin of this large change in $I_{p}$ (and thus $P_{s}$ ) by first noting that it primarily occurs below the FM ordering temperature, $T_{\mathrm{C}} \sim 240 \mathrm{~K}$ (Fig. $3 \mathrm{~b}$ ), indicating that FM order in LCMO is linked to the observed $\Delta I_{p}$. In addition, the timescale is comparable to that for $s-1$ relaxation in LCMO, as determined through comparison of our TR-SHG data to optical pump-probe measurements on our BSTO/LCMO heterostructure (Fig. 4). This suggests that the observed photoinduced ME response is initiated by a change in the lattice constant through magnetostriction ${ }^{45}$, which arises from photoinduced demagnetization of LCMO. More specifically, s-1 relaxation reduces the magnetization in the FM-ordered state, gradually decreasing the magnitude of the existing in-plane lattice contraction due to spin-spin interactions and applying stress to BSTO (Fig. 2a, $t \sim 50 \mathrm{ps}$ ); more detail on general magnetostriction is given in Supplementary Note 2. Then, as for the early time dynamics, the resulting increase in the in-plane tensile stress on BSTO initiates a piezoelectric effect along the $z$ direction (Fig. 1c), reducing the dielectric displacement $D_{z}$ and modifying the FE polarization $P_{\mathrm{s}}$ (Fig. 2a).

Additional experimental evidence enables us to rule out other possibilities that could lead to the observed change in $\Delta I_{p}$. First,
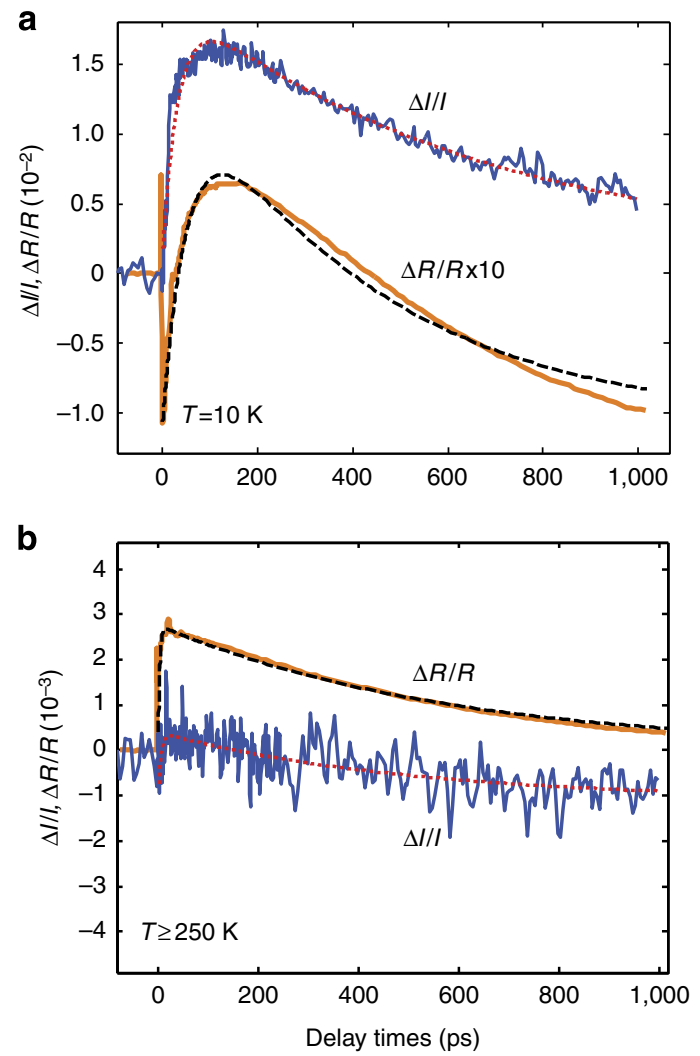

Figure 4 | Comparison of $\Delta \boldsymbol{R} / \boldsymbol{R}$ and $\boldsymbol{\Delta} \boldsymbol{I} / \mathbf{I}$. Comparison at (a) $10 \mathrm{~K}$ and (b) above $250 \mathrm{~K} . \Delta R / R$ is measured with a $p$-polarized probe and $\Delta I / I$ is taken in the $(p, p)$ configuration. The solid lines are the experimental data and the dashed lines represent a double exponential fit. Additional discussion of the comparison between the $\Delta R / R$ and $\Delta l / I$ data is given in Methods and Supplementary Note 1.
$\Delta I_{p}$ at low temperatures does not originate from a photoinduced modulation in the reflectivity due to changes in the optical constants; $\Delta R / R$ is an order of magnitude smaller than $\Delta I / I$ (the normalized photoinduced change in SHG intensity for a given polarization configuration) at low temperatures (Fig. 4), and unlike $\Delta I / I$, it does not vary with the incident probe polarization (Supplementary Fig. 1). Second, there is no observable SHG signal, either static $\left(I_{p}\right)$ or photoinduced $(\Delta I / I)$, from a single LCMO film, verifying that $\Delta I_{p}$ in our BSTO/LCMO heterostructure does not result from SHG in LCMO. Third, there is no measurable $\Delta I / I$ signal from a single BSTO film upon $1.59 \mathrm{eV}$ excitation, since the pump is far below the gap ${ }^{33}$. Fourth, we note that the observed features are independent of the pump polarization, consistent with photoinduced demagnetization in LCMO. Fifth, Kerr rotation of the reflected light at both 1.55 and $3.1 \mathrm{eV}$, induced by demagnetization in LCMO, does not contribute to $\Delta I_{p}$. Previous work has shown that the Kerr rotation in manganites for similar pump fluences is $\sim 0.01-0.1$ degrees $^{44,46}$. This small rotation is unlikely to result in the large changes in SHG intensity that we observed. In addition, if it were to contribute significantly to $\Delta I / I$, it would also be observed in TR-SHG measurements on a single LCMO film, where Kerr rotation dominates polarization-dependent measurements ${ }^{44,46}$. Moreover, in the unlikely case that Kerr rotation in this heterostructure is larger than that of a single LCMO layer upon photoexcitation, the rotated fundamental or SHG polarizations would induce phase shifts in our polarization-dependent TR-SHG measurements, which are not observed (Figs $2 \mathrm{~b}$ and 3 ).

Finally, any contributions from surface SHG, including any potential time-dependent interference with the contribution from FE order, are expected to be negligible. The temperaturedependent static SHG signal (Fig. 1d) originates primarily from bulk FE order in BSTO $^{33}$. Surface and interface contributions from this layer have the same symmetry, $C_{4 \mathrm{v}}$, as the bulk FE order. Any interference between these contributions will be either in-phase or $180^{\circ}$ out-of-phase, but will remain constant over the entire sample, as a spatially homogeneous SHG intensity is observed after SHG saturation ${ }^{33}$. Well above $T_{\mathrm{FE}}$, the residual SHG signal likely originates from the surface or interface and mixes with other potential contributions, such as slow carrier transfer $^{33}$ and size effects ${ }^{37,47}$ in thin films. Upon photoexcitation, surface SHG does not contribute to the $\Delta I / I$ signal, since the BSTO surface is not excited. Furthermore, we can exclude any contribution from changes in interfacial SHG due to demagnetization of LCMO, because no TR-SHG signal was obtained from the interface between LCMO and the $\mathrm{MgO}$ substrate. These considerations allow us to conclude that the $\Delta I / I$ signal from our heterostructure originates from changes in $P_{s}$ that are initiated by photoinduced changes in LCMO.

\section{Discussion}

It is worth re-emphasizing that, as discussed in the introduction, the photoinduced ME response measured here is analogous to the ME effect observed when an external B field is applied to enhance the lattice contraction through magnetostriction in a FE/FM bilayer, inducing a piezoelectric response in the FE layer ${ }^{14}$. The major advantage of our approach lies in the temporal degree of freedom offered by femtosecond laser pulses, enabling us to optically manipulate the $\mathrm{ME}$ response in the time domain and also discriminate between different contributions to the ME response. For example, we can temporally separate the comparatively small changes in $P_{s}$ at early times due to heatinginduced expansion of LCMO (Fig. 2, $t \sim 1 \mathrm{ps)}$ from the larger changes in $P_{s}$ on longer timescales due to the reduction in the LCMO lattice contraction by magnetostriction (Fig. $2, t \sim 50 \mathrm{ps)}$. 
The difference in the magnitude of these changes is likely due to the strong interaction between the ordered spins in LCMO (causing the lattice to contract through magnetostriction), which only permits a small amount of heating-induced thermal stress at early times. As a result, the in-plane tensile stress does not significantly increase before $s-1$ relaxation takes place. Therefore, strain relaxation throughout the entire BSTO film only occurs after tens of ps, setting the timescale for the maximum changes in FE polarization (Fig. 3a). Finally, we note that at long (hundreds of ps to ns) timescales, the remaining changes in the FE polarization are due to thermal diffusion from LCMO to BSTO.

In conclusion, we used femtosecond optical pulses to manipulate the coupling between FE and FM order in a complex oxide heterostructure, employing TR-SHG to optically perturb the magnetization in LCMO and selectively probe the associated change in the FE properties of BSTO. We find that, although lattice heating in LCMO occurs within $\sim 1 \mathrm{ps}$, the resulting lateral stress on BSTO is relatively small compared to the strong lattice contraction originating from spin-spin interactions in LCMO. This lattice contraction is reduced through spin-lattice relaxation, increasing the tensile stress on BSTO through magnetostriction in LCMO and leading to a piezoelectric response that changes the FE polarization. Therefore, coupling between FE and FM order in $\mathrm{BSTO} / \mathrm{LCMO}$ is induced within tens of picoseconds, mediated through elastic coupling between the BSTO and LCMO layers. Femtosecond optical spectroscopy can thus shed light on the mechanisms underlying ME coupling in complex oxide heterostructures, while presenting intriguing possibilities for future high-speed optically controlled ME devices (for example, through coherent spin control ${ }^{48-50}$, which would enable faster manipulation of spin dynamics and minimize undesired heat flow in device applications.)

\section{Methods \\ Sample fabrication and characterization. The samples used in our studies are 50-nm-thick BSTO films grown by pulsed laser deposition on 50-nm-thick optimally doped manganite films (LCMO), using (100) MgO substrates. The substrate temperature during film growth is initially optimized and maintained at $750{ }^{\circ} \mathrm{C}$. The oxygen pressure during deposition is $100 \mathrm{mTorr}$. The samples are cooled to room temperature in pure oxygen (at a pressure of 350 Torr) without further thermal treatment. X-ray diffraction measurements reveal a compressive strain in epitaxially grown BSTO, while LCMO on $\mathrm{MgO}$ is relaxed. This causes an increase in tetragonality, even at room temperature, enhancing the FE $T_{\mathrm{C}}$ to $\sim 215 \mathrm{~K}$, which is significantly higher than the bulk $T_{\mathrm{C}} \sim 75 \mathrm{~K}$. More detailed characterization of these samples is discussed in ref. 33.}

TR-SHG experimental details. Our TR-SHG experiments are based on an amplified Ti:sapphire laser system producing pulses at a $250-\mathrm{kHz}$ repetition rate with $\sim 100$-fs duration and energies of $\sim 4 \mu \mathrm{J}$ at a centre wavelength of $780 \mathrm{~nm}$ $(1.59 \mathrm{eV})$. SHG is generated from BSTO at $3.18 \mathrm{eV}$ using a $1.59-\mathrm{eV}$ probe beam, after which it is detected by a photomultiplier tube using lock-in detection after filtering out the fundamental signal. The fundamental light polarization is controlled by a half-wave plate ( $\lambda / 2$ in Fig. 1a) and the SHG signal is detected for either $p$ or $s$ polarizations. The standard laser fluence used for SHG in our measurements is $F_{0} \sim 0.25 \mathrm{~mJ} \mathrm{~cm}^{-2}$ and the pump fluence is $\sim 1 \mathrm{~mJ} \mathrm{~cm}^{-2}$, creating a carrier density of $\sim 10^{20} \mathrm{~cm}^{-3}\left(\sim 10^{-2}\right.$ per unit cell). In the $C_{4 \mathrm{v}}$ symmetry, SHG can be generated and probed when the fundamental light is incident at an angle away from the sample normal, thus exhibiting a sinusoidal dependence on the incident angle $\theta$ (as observed in separate angle-dependent measurements on our samples). Therefore, we used a reflection geometry in which the fundamental light was incident at a $\sim 20^{\circ}$ angle to obtain the data shown in our manuscript. The pump beam was incident at a smaller angle of $\sim 10-15^{\circ}$, and its specular reflection was completely separated from the probe beam path. The temporal delay is achieved by a mechanical delay stage that allows us to vary the probe path length. Finally, ref. 33 gives a detailed static SHG characterization of these samples. Here, all timeresolved data were taken after saturation of the static SHG signal ${ }^{33}$.

SHG analysis. For the third-rank tensor $d_{i j k}$, the indices $j k$ can be replaced by $l=1,2, \ldots, 6$, corresponding to $x x, y y, z z, y z, x z$ and $x y$, respectively, using simple symmetry arguments that reduce 27 tensor components to 18 . Further reduction can be achieved by noting that the $C_{4 v}$ symmetry has $d_{31}=d_{z x x}=d_{32}=d_{z y y}$, $d_{15}=d_{x x z}=d_{24}=d_{y y z}$ and $d_{33}=d_{z z z}$. Therefore, we use the three independent indices 15,31 and 33 here. To represent the fourth-rank stress tensor $e_{i j k}$, the first $i=1,2, \ldots, 6$ as defined above, and $j, k=1,2,3$ correspond to the Cartesian coordinate $x, y, z$, respectively. It also can be reduced according to the above discussion of indices $j, k$.

Figure $1 \mathrm{~b}$ depicts the static SHG signals for $p$ and $s$ polarizations, $I_{p}$ and $I_{s}$. In reflection (Fig. 1a), $\phi$ determines the polarization of the fundamental light that generates SHG. With a unit $E$ field of the fundamental light incident at an angle $\theta$ (Fig. 1a), we have components $\left(E_{x}(\omega), E_{y}(\omega), E_{z}(\omega)\right)=(\cos \phi \cos \theta, \sin \phi,-\cos \phi$ $\sin \theta$ ), where $\theta=0$ is along $z$ and $\phi=0^{\circ}$ and $90^{\circ}$ represent $p$ and $s$ polarizations of the incident fundamental light, respectively. When the detection polarizer is set to $p$ or $s$, we detect the SHG dipole components $\left(P_{x}(2 \omega), P_{y}(2 \omega), P_{z}(2 \omega)\right)$ along $(1,0,1)$ and $(0,1,0)$, respectively, with each component linked to $\left(d_{15}=d_{24}\right.$, $\left.d_{31}=d_{32}, d_{33}\right)$ by

$$
\begin{gathered}
P_{x}(2 \omega)=2 \epsilon_{0} d_{15} E_{x}(\omega) E_{z}(\omega), \\
P_{y}(2 \omega)=2 \epsilon_{0} d_{15} E_{y}(\omega) E_{z}(\omega), \\
P_{z}(2 \omega)=\epsilon_{0}\left(d_{31} E_{x}^{2}(\omega)+d_{31} E_{y}^{2}(\omega)+d_{33} E_{z}^{2}(\omega)\right) .
\end{gathered}
$$

We thus can derive the SHG intensities $I_{p}$ and $I_{s}$ as functions of $\phi$ (Fig. 1b):

$$
\begin{gathered}
I_{p}=\left(a \cos ^{2} \phi+b \sin ^{2} \phi\right)^{2}, \\
I_{s}=(c \sin (2 \phi))^{2},
\end{gathered}
$$

where $a, b$ and $c$ are functions of $\theta$ and linear combinations of $d_{15}, d_{31}$ and $d_{33}$. This enables us to detect different components of $d_{i j k}$ through different polarization combinations of (incident light, SHG). The combination of $\left(45^{\circ}, s\right)$ probes $c$, associated with $d_{15}$, while $(s, p)$ detects $b$, associated with $d_{31}$. The $(p, p)$ configuration measures $a$ as a linear combination of all three independent components of $d_{i j k}$, allowing us to extract $d_{33}$ (Supplementary Table 1) from the measurements in the $\left(45^{\circ}, s\right)$ and $(s, p)$ configurations.

We can use the above analysis methods to numerically fit our TR-SHG data (Fig. 3a). To do this, we need to know the new values of $a$ and $b$ after photoexcitation, which can then be inserted into equation (4). First, we note that the polarization dependence of $\Delta I_{p}$ for $t>0$ (that is, $\Delta I_{p}$ decreases for $(s, p)$ and increases for $(p, p)$ ) (Fig. 3) implies that a decrease in $b$ is accompanied by an increase in $a$. The TR-SHG signal in the $(s, p)$ configuration is solely due to $b$ (originating from $d_{31}$ (equation (4))), which has the opposite sign of $a$ (Supplementary Table 1). Therefore, the photoinduced decrease in $d_{31}$ implies an increase in $a$, as observed for the $(p, p)$ configuration in Fig. 3. Next, we consider the coefficient $c$, which solely originates from $d_{15}$. There are three significant features we notice from the photoinduced change in $d_{15}$. First, $\Delta I / I$ is much smaller than that measured for the $(p, p)$ and $(s, p)$ configurations (Supplementary Fig. 1). Second, the change is comparable to the modulation arising from the change in optical constants (Supplementary Fig. 1c; Fig. 4). Third, it does not have significant temperature dependence, unlike the other two polar combinations (Supplementary Fig. 1).

We conclude that $d_{15}$ has an insignificant contribution to $a$, and the photoinduced changes in $d_{15}$ could be strongly influenced by photoinduced reflectivity changes in BSTO. These considerations allowed us to simulate the photoinduced changes in $d_{33}$ and $d_{31}$, revealing that $\Delta d_{33} / d_{33} \sim-4 \%$ and $\Delta d_{31} / d_{31} \sim-6 \%$. We thus can use these reductions in $d_{31}$ and $d_{33}$, together with the isotropic reduction of $d_{31}, d_{33}$ and $d_{15}$ due to residual heating, to numerically fit $\Delta I_{p}$ (dashed lines in Fig. 3a). These transient changes lead to the feature observed in $I_{p}$ after $t_{0}=0$ (Fig. 3a,b)

\section{References}

1. Eerenstein, W., Mathur, N. D. \& Scott, J. F. Multi-ferroic and magnetoelectric materials. Nature 442, 759-765 (2006).

2. Khomskii, D. Classifying multiferroics: mechanisms and effects. Physics 2, 20 (2009).

3. Cheong, S.-W. \& Mostovoy, M. Multiferroics: a magnetic twist for ferroelectricity. Nat. Mater. 6, 13-20 (2007).

4. Ramesh, R. \& Spaldin, N. A. Multiferroics: progress and prospects in thin films Nat. Mater. 6, 21-29 (2007).

5. Vaz, C. A. F., Hoffman, J., Ahn, C. H. \& Ramesh, R. Magnetoelectric coupling effects in multiferroic complex oxide composite structures. Adv. Mater. 22, 2900-2918 (2010).

6. Zheng, H. et al. Multiferroic $\mathrm{BaTiO}_{3}-\mathrm{CoFe}_{2} \mathrm{O}_{4}$ nanostructures. Science 303, 661-663 (2004).

7. Scott, J. F. Applications of modern ferroelectrics. Science 315, 954-959 (2007).

8. Garcia, V. et al. Ferroelectric control of spin polarization. Science 327, 1106-1110 (2010).

9. Buzzi, M. et al. Single domain spin manipulation by electric fields in strain coupled artificial multiferroic nanostructures. Phys. Rev. Lett. 111, 027204 (2013). 
10. Ma, J., Hu, J., Li, Z. \& Nan, C.-W. Recent progress in multiferroic magnetoelectric composites: from bulk to thin films. Adv. Mater. 23, 1062-1087 (2011).

11. van Suchtelen, J. Product properties: a new application of composite materials. Philips Res. Rep. 27, 28 (1972).

12. Nan, C.-W., Bichurin, M. I., Dong, S., Viehland, D. \& Srinivasan, G. Multiferroic magnetoelectric composites: Historical perspective, status, and future directions. J. Appl. Phys. 103, 031101 (2008).

13. Fiebig, M. \& Spaldin, N. A. Current trends of the magnetoelectric effect. Eur. Phys. J. B 71, 293-297 (2009).

14. Srinivasan, G., Rasmussen, E. T., Levin, B. J. \& Hayes, R. Magnetoelectric effects in bilayers and multilayers of magnetostrictive and piezoelectric perovskite oxides. Phys. Rev. B 65, 134402 (2002).

15. Kambale, R. C., Jeong, D.-Y. \& Ryu, J. Current status of magnetoelectric composite thin/thick films. Adv. Condens. Matter Phys. 2012, 824643 (2012)

16. Sheu, Y. M. et al. Ultrafast carrier dynamics and radiative recombination in multiferroic $\mathrm{BiFeO}_{3}$. Appl. Phys. Lett. 100, 242904 (2012).

17. Wen, H. et al. Electronic origin of ultrafast photoinduced strain in $\mathrm{BiFeO}_{3}$. Phys. Rev. Lett. 110, 037601 (2013).

18. Matsubara, M., Kaneko, Y., He, J.-P., Okamoto, H. \& Tokura, Y. Ultrafast polarization and magnetization response of multiferroic $\mathrm{GaFeO} 3$ using time-resolved nonlinear optical techniques. Phys. Rev. B 79, 140411 (2009).

19. Ogawa, N., Satoh, T., Ogimoto, Y. \& Miyano, K. Half-metallic spin dynamics at a single $\mathrm{LaMnO}_{3} / \mathrm{SrMnO}_{3}$ interface studied with nonlinear magneto-optical Kerr effect. Phys. Rev. B 80, 241104 (2009).

20. Fiebig, M. et al. Ultrafast magnetization dynamics of antiferromagnetic compounds. J. Phys. D Appl. Phys. 41, 164005 (2008).

21. Wang, Y. T., Luo, C. W. \& Kobayashi, T. Understanding multiferroic hexagonal manganites by static and ultrafast optical spectroscopy. Adv. Condens. Matter Phys. 2013, 104806 (2013)

22. Hoffmann, T., Thielen, P., Becker, P., Bohatý, L. \& Fiebig, M. Time-resolved imaging of magnetoelectric switching in multiferroic $\mathrm{MnWO}_{4}$. Phys. Rev. B 84, 184404 (2011).

23. Jones, S. P. P. et al. High-temperature electromagnons in the magnetically induced multiferroic cupric oxide driven by intersublattice exchange. Nat. Commun. 5, 3787 (2014).

24. Jang, K.-J. et al. Ultrafast IR spectroscopic study of coherent phonons and dynamic spin-lattice coupling in multiferroic $\mathrm{LuMnO}_{3}$. New J. Phys. 12, 023017 (2010).

25. Talbayev, D. et al. Detection of coherent magnons via ultrafast pump-probe reflectance spectroscopy in multiferroic $\mathrm{Ba}_{0.6} \mathrm{Sr}_{1.4} \mathrm{Zn}_{2} \mathrm{Fe}_{12} \mathrm{O}_{22}$. Phys. Rev. Lett. 101, 097603 (2008)

26. Kimel, A. V., Pisarev, R. V., Bentivegna, F. \& Rasing, T. h. Time-resolved nonlinear optical spectroscopy of $\mathrm{Mn}^{3+}$ ions in rare-earth hexagonal manganites $R \mathrm{MnO}_{3}(R=\mathrm{Sc}, \mathrm{Y}, \mathrm{Er})$. Phys. Rev. B 64, 201103 (2001).

27. Sheu, Y. M. et al. Polaronic transport induced by competing interfacial magnetic order in a $\mathrm{La}_{0.7} \mathrm{C}_{0.3} \mathrm{MnO}_{3} / \mathrm{BiFeO}_{3}$ heterostructure. Phys. Rev. X 4, 021001 (2014).

28. Rana, D. S. et al. Understanding the nature of ultrafast polarization dynamics of ferroelectric memory in the multiferroic BiFeO3. Adv. Mater. 21, 2881-2885 (2009).

29. Shih, H. C. et al. Magnetization dynamics and the $\mathrm{Mn}^{3+} \mathrm{d}-\mathrm{d}$ excitation of hexagonal $\mathrm{HoMnO}_{3}$ single crystals using wavelength-tunable time-resolved femtosecond spectroscopy. Phys. Rev. B 80, 024427 (2009).

30. Handayani, I. P. et al. Dynamics of photoexcited electrons in magnetically ordered $\mathrm{TbMnO}_{3}$. J. Phys. Condens. Matter 25, 116007 (2013).

31. Qi, J. et al. Coexistence of coupled magnetic phases in epitaxial $\mathrm{TbMnO}_{3}$ films revealed by ultrafast optical spectroscopy. Appl. Phys. Lett. 101, 122904 (2012).

32. Ogawa, N., Ogimoto, Y. \& Miyano, K. Ultrafast dynamics of orbital-orderinduced polarization. Appl. Phys. Lett. 102, 251911 (2013).

33. Sheu, Y. M. et al. Photoinduced stabilization and enhancement of the ferroelectric polarization in $\mathrm{Ba}_{0.1} \mathrm{Sr}_{0.9} \mathrm{TiO}_{3} / \mathrm{La}_{0.7} \mathrm{Ca}(\mathrm{Sr})_{0.3} \mathrm{MnO}_{3}$ thin film heterostructures. Phys. Rev. B 88, 020101 (2013).

34. Pertsev, N. A., Zembilgotov, A. G. \& Tagantsev, A. K. Effect of mechanical boundary conditions on phase diagrams of epitaxial ferroelectric thin films. Phys. Rev. Lett. 80, 1988-1991 (1998).

35. Bristowe, N. C., Stengel, M., Littlewood, P. B., Pruneda, J. M. \& Artacho, E. Electrochemical ferroelectric switching: origin of polarization reversal in ultrathin films. Phys. Rev. B 85, 024106 (2012).
36. Stefanita, C.-G. From Bulk to Nano (Springer, 2008).

37. Pugachev, A. M. et al. Broken local symmetry in paraelectric $\mathrm{BaTiO}_{3}$ proved by second harmonic generation. Phys. Rev. Lett. 108, 247601 (2012).

38. Miller, R. C. Optical second harmonic generation in piezoelectric crystals. Appl. Phys. Lett. 5, 17-19 (1964).

39. Denev, S. A., Lummen, T. T. A., Barnes, E., Kumar, A. \& Gopalan, V. Probing ferroelectrics using optical second harmonic generation. J. Am. Ceram. Soc. 94, 2699 (2011).

40. Fiebig, M., Pavlov, V. V. \& Pisarev, R. V. Second-harmonic generation as a tool for studying electronic and magnetic structures of crystals: review. J. Opt. Soc Am. B 22, 96-118 (2005).

41. Fiebig, M., Lottermoser, T. h., Fröhlich, D., Goltsev, A. V. \& Pisarev, R. V. Observation of coupled magnetic and electric domains. Nature 419, 818-820 (2002).

42. Averitt, R. D. \& Taylor, A. J. Ultrafast optical and far-infrared quasiparticle dynamics in correlated electron materials. J. Phys. Condens. Matter 14, R1357 (2002).

43. Müller, G. M. et al. Spin polarization in half-metals probed by femtosecond spin excitation. Nat. Mater. 8, 56-61 (2009).

44. Ogasawara, T. et al. Photoinduced spin dynamics in $\mathrm{La}_{0.6} \mathrm{Sr}_{0.4} \mathrm{MnO}_{3}$ observed by time-resolved magneto-optical Kerr spectroscopy. Phys. Rev. B 68, 180407 (2003).

45. Radaelli, P. G. et al. Simultaneous structural, magnetic, and electronic transitions in $\mathrm{La}_{1-x} \mathrm{Ca}_{x} \mathrm{MnO}_{3}$ with $\mathrm{x}=0.25$ and 0.50. Phys. Rev. Lett. 75, 4488-4491 (1995).

46. McGill, S. A. et al. Optical evidence for transient photoinduced magnetization in $\mathrm{La}_{0.7} \mathrm{Ca}_{0.3} \mathrm{MnO}_{3}$. Phys. Rev. B 71, 075117 (2005).

47. Choi, K. J. et al. Enhancement of ferroelectricity in strained $\mathrm{BaTiO}_{3}$ thin films. Science 306, 1005-1009 (2004).

48. Kimel, A. V. et al. Ultrafast nonthermal control of magnetization by instantaneous photomagnetic pulses. Nature 435, 655-657 (2005).

49. Kirilyuk, A., Kimel, A. V. \& Rasing, T. h. Ultrafast optical manipulation of magnetic order. Rev. Mod. Phys. 82, 2731-2784 (2010).

50. Bossini, D., Kalashnikova, A. M., Pisarev, R. V., Rasing, T. h. \& Kimel, A. V. Controlling coherent and incoherent spin dynamics by steering the photoinduced energy flow. Phys. Rev. B 89, 060405 (2014).

\section{Acknowledgements}

This work was performed at the Center for Integrated Nanotechnologies, a US Department of Energy, Office of Basic Energy Sciences user facility. Partial support was also provided by Los Alamos National Laboratory's Directed Research and Development program. Los Alamos National Laboratory, an affirmative action equal opportunity employer, is operated by Los Alamos National Security, LLC, for the National Nuclear Security Administration of the US Department of Energy under Contract No. DE-AC52 06NA25396.

\section{Author contributions}

Y.M.S. designed and carried out the experiments and data analysis, with input from R.P.P. and A.J.T. Y.M.S. and S.A.T. performed the theoretical analysis and physical interpretation of the data, with additional input from R.P.P. Y.M.S. and R.P.P. wrote the manuscript, with additional input from all coauthors. R.P.P. and A.J.T. directed the project. L.Y. and Q.X.J. prepared the samples for the experiments.

\section{Additional information}

Supplementary Information accompanies this paper at http://www.nature.com/ naturecommunications

Competing financial interests: The authors declare no competing financial interests

Reprints and permission information is available online at http://npg.nature.com/ reprintsandpermissions/

How to cite this article: Sheu, Y. M. et al. Using ultrashort optical pulses to couple ferroelectric and ferromagnetic order in an oxide heterostructure. Nat. Commun. 5:5832 doi: $10.1038 /$ ncomms6832 (2014) 